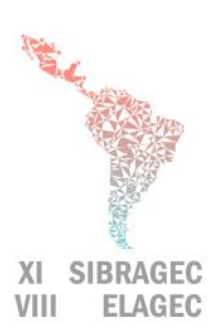

XI SIMPÓSIO BRASILEIRO DE GESTÃO E ECONOMIA DA CONSTRUÇÃO

VIII ENCUENTRO LATINOAMERICANO DE GESTIÓN Y ECONOMÍA DE LA CONSTRUCCIÓN

Del conocimiento a la acción: prácticas avanzadas de gestión de la producción

Londrina, Paraná, Brasil. 23 al 25 de Octubre del 2019

\title{
LAS PRÁCTICAS LEAN Y SU APORTE A LA DISMINUCIÓN DE IMPACTOS AMBIENTALES DEL SECTOR DE LA CONSTRUCCIÓN
}

\author{
BAHAMÓN-JARAMILLO, Sara (1); BOTERO BOTERO, Luis Fernando (2); \\ VÁSQUEZ-HERNÁNDEZ, Alejandro (3)
}

(1) Universidad Eafit, teléfono: (57) 4 2619500, e-mail: sbahamon@eafit.edu.co (2) Universidad Eafit, teléfono: (57) 4 2619500, e-mail: 1fbotero@eafit.edu.co, (3) Universidad Eafit, teléfono: (57) 4 2619500, e-mail: avasqu12@eafit.edu.co

\begin{abstract}
Lean and Sustainable Construction are two philosophies that share the purpose of efficient use of resources. However, they are mostly applied individually without considering the integration of both in a Construction project. This study seeks to identify the environmental impacts of the most usual Construction activities and Lean Construction practices that help reduce, mitigate or prevent them. For this, an interaction matrix was developed to identify the environmental impacts associated with each Construction activity considered and subsequently, a detailed literature review was carried out looking for Lean practices that could contribute to the prevention or reduction of these impacts. The generation of ordinary, recyclable, hazardous and $C \& D$ wastes are the environmental impacts with the greatest number of Lean Construction practices that can help reduce or prevent them. Of the 13 Lean Construction practices identified, prefabricated Construction is the practice that may contribute to the reduction or prevention of the majority of the environmental impacts (12 of them).
\end{abstract}

Keywords: Lean Construction, Sustainable Construction, environmental impacts, Lean practices

\section{INTRODUCCIÓN}

La industria de la construcción se caracteriza por su alto consumo de recursos naturales, alta generación de desperdicios, alta producción de emisiones y su potencial de degradación ambiental (BANAWI; BILEC, 2014; VASCONCELOS et al., 2015). Se considera como una de las industrias más contribuyentes a los impactos ambientales globales, lo que ha llevado a que adquiera una gran responsabilidad en la búsqueda de edificaciones comprometidas con el medio ambiente, bajo el lineamiento de estrategias sostenibles (DE CARVALHO; GRANJA; DA SILVA, 2017; GOLZARPOOR et al., 2017). La construcción está en la necesidad de una mejor eficiencia de recursos, y a su vez, de aumentar su productividad, generar menos desperdicios e incrementar el valor de sus productos (JOHNSEN; DREVLAND, 2016). Lean Construction (LC) se presenta como guía referente, puede ayudar a generar cambios significativos, debido a su gran potencial de incrementar el valor para los clientes y disminuir todos los tipos de desperdicios (M. S. BAJJOU, A. CHAFI, 2017).

BAHAMÓN-JARAMILLO, S.; BOTERO BOTERO, L. F.; VÁSQUEZ-HERNÁNDEZ, A. Las prácticas lean y su aporte a la disminución de impactos ambientales del sector de la construcción. In: SIMPÓSIO BRASILEIRO DE GESTÃO E ECONOMIA DA CONSTRUÇÃO, 11., 2019, Londrina. Anais [...].

Porto Alegre: ANTAC, 2019. Disponível em:

https://www.antaceventos.net.br/index.php/sibragec/sibragec2019/paper/view/401 
LC es considerada como una oportunidad para reducir los impactos ambientales de la construcción (SAGGIN et al., 2015). Según la Agencia de Protección Ambiental (EPA, 2000), Lean propicia un ambiente estratégico y cultural favorable para prevenir la contaminación y reducir las cantidades de desechos, promoviendo la sostenibilidad. Si bien la Construcción Sostenible y LC son dos enfoques que tienen como fin la eficiencia del uso de los recursos, son aplicados mayormente de manera individual sin considerar la integración de ambos en un proyecto de construcción (PULASKI et al., 2004).

Con base en esto, este trabajo busca identificar los impactos ambientales generados por las actividades de construcción más usuales y las prácticas de LC que puedan favorecer a la disminución, mitigación o prevención de dichos impactos.

\section{LEAN CONSTRUCTION, CONSTRUCCIÓN SOSTENIBLE Y SU TRASCENDENCIA EN LOS IMPACTOS AMBIENTALES}

La Comisión Mundial sobre Medio Ambiente y Desarrollo, en el año 1987, propuso el término de sostenibilidad como respuesta a los impactos de las intervenciones hechas por el hombre al medio ambiente (SAIEG et al., 2018). En el sector de la construcción, la sostenibilidad puede contribuir a problemas globales si se reconsideran los procesos en cuanto al uso de materiales, de energía, contaminación y residuos de construcción y demolición (RCDs) (AHUJA, 2013). Algunos autores mencionan que "La Construcción Lean ofrece la base conceptual y el potencial para la Construcción Sostenible" (SALEM et al., 2014).

LC puede ayudar a alcanzar la sostenibilidad en sus tres dimensiones: socialmente, gestionando la seguridad, salud, el entorno laboral y la lealtad entre los involucrados; economicamente, mediante la disminución de costos y recursos; y ambientalmente reduciendo los desperdicios, disminuyendo la contaminación y ayudando a preservar los recursos. (JOHNSEN; DREVLAND, 2016; KHODEIR; OTHMAN, 2016). Simultáneamente, recientes investigaciones hacen referencia a que, mediante las herramientas Lean es viable alcanzar beneficios ambientales, siempre y cuando se reduzca la generación de desperdicios, haya prevención de contaminación del aire, auditiva y se aumente el valor para los clientes (CARNEIRO et al., 2012).

\section{MÉTODO DE INVESTIGACIÓN}

El proceso para la identificación, valoración y mitigación de los impactos ambientales se conoce como la evaluación de impacto ambiental (EIA) (SENÉCAL et al., 1999). Para la caracterización de estos impactos, se reconocen los problemas generados durante la ejecución de los procesos constructivos, a través de la metodología de una matriz de interacción. Para la valoración y evaluación, diversos autores han planteado sus propios métodos, desde (LEOPOLD et al., 1971) hasta (CONESA FERNÁNDEZ-VITORA, 2009).

Con el fin de identificar los impactos ambientales de las actividades constructivas más habituales, se realizó la Tabla 1 utilizando el método Matriz de Interacción. En esta, se consideraron 29 actividades que se correlacionan con los 21 impactos que generan.

Luego, para conocer las prácticas Lean que pueden contribuir a la disminución o mitigación de estos impactos, se hizo una revisión detallada de la literatura, en la que, diferentes autores coinciden en señalar, cuales prácticas le aportan a la prevención o disminución de dichos impactos; ver Tabla 2. 
SIBRAGEC - ELAGEC 2019 - del 23 al 25 de Octubre - LONDRINA - PR

\section{RESULTADOS Y ANÁLISIS DE INVESTIGACIÓN}

\subsection{Impactos ambientales del sector constructor}

El sector de la construcción consume grandes cantidades de recursos y a su vez origina grandes cantidades de desechos (BANAWI; BILEC, 2014), generando efectos negativos, que se transforman en impactos al medio ambiente (DE CARVALHO; GRANJA; DA SILVA, 2017; JOHNSEN; DREVLAND, 2016). Estos impactos van desde residuos, contaminación del aire, del agua, hasta el cambio climático, remoción de vegetación, emisiones peligrosas, entre otros. (ENSHASSI; KOCHENDOERFER; RIZQ, 2014). La Tabla 1, presenta la matriz de interacción entre cada actividad de construcción y sus respectivos impactos ambientales asociados. Esta fue construida a partir de los parámetros propuestos por $\left(360^{\circ}\right.$ EN CONCRETO, 2013; AMBIENTE, 2013; CALDERÓN， 2009; ENSHASSI; KOCHENDOERFER; RIZQ， 2014; GONZÁLEZ, 2008).

Tabla 1 - Impactos ambientales con relación a las actividades de construcción

\begin{tabular}{|c|c|c|c|c|c|c|c|c|c|c|c|c|c|}
\hline \multirow{2}{*}{$\begin{array}{l}\text { Ampactos } \\
\begin{array}{l}\text { Actividades de } \\
\text { construcción }\end{array}\end{array}$} & \multicolumn{7}{|c|}{ Generación } & \multicolumn{4}{|c|}{ Consumo } & \multicolumn{2}{|c|}{ Emisión } \\
\hline & 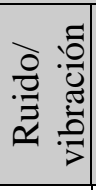 & 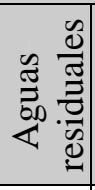 & 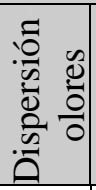 & $\underset{\vartheta}{\vartheta}$ & 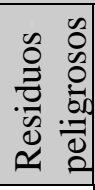 & 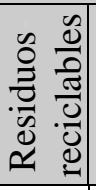 & 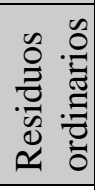 & 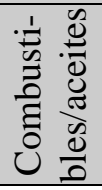 & $\frac{\tilde{d}}{\frac{\pi}{\pi}}$ & 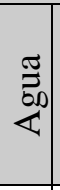 & 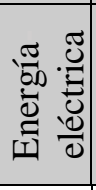 & 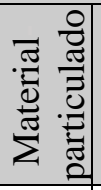 & $\underset{\mathbb{E}}{\mathbb{E}}$ \\
\hline Demolición & $\mathrm{X}$ & $\mathrm{X}$ & & $\mathrm{x}$ & $\mathrm{x}$ & & & & & & & $\mathrm{X}$ & $\mathrm{x}$ \\
\hline Adecuación terreno & $\mathrm{X}$ & & & & $\mathrm{X}$ & $\mathrm{X}$ & $\mathrm{x}$ & & & & & & \\
\hline Adecuaciones provisionales & & & $\mathrm{X}$ & & $\mathrm{X}$ & & & & & & & & \\
\hline Excavación & $\mathrm{x}$ & $\mathrm{x}$ & & & $\mathrm{X}$ & $\mathrm{x}$ & & & & & & $\mathrm{x}$ & $\mathrm{X}$ \\
\hline Descapote vegetación & $\mathrm{x}$ & & & & $\mathrm{X}$ & $\mathrm{x}$ & $\mathrm{x}$ & & & & & & $\mathrm{X}$ \\
\hline Corte/compactación terreno & $\mathrm{x}$ & $\mathrm{x}$ & & $\mathrm{x}$ & $\mathrm{x}$ & $\mathrm{x}$ & & & & & & & $\mathrm{x}$ \\
\hline Uso explosivos & $\mathrm{x}$ & $\mathrm{x}$ & & & & & & & & & & $\mathrm{x}$ & \\
\hline Preparación concreto & $\mathrm{x}$ & $\mathrm{x}$ & $\mathrm{X}$ & $\mathrm{x}$ & $\mathrm{x}$ & & & & & $\mathrm{x}$ & $\mathrm{x}$ & $\mathrm{x}$ & $\mathrm{X}$ \\
\hline Fundido concreto & & $\mathrm{x}$ & & & $\mathrm{X}$ & & & & & & & & \\
\hline Curado concreto & & & $\mathrm{X}$ & $\mathrm{x}$ & & & & & & & & & \\
\hline Armado encofrado & & $\mathrm{x}$ & $\mathrm{X}$ & & & & & & & & & & \\
\hline Impermeabilización & & $\mathrm{X}$ & $\mathrm{X}$ & & & & & & & & & & \\
\hline Preparación mortero & $\mathrm{x}$ & $\mathrm{x}$ & $\mathrm{X}$ & $\mathrm{X}$ & $\mathrm{X}$ & & & & & $\mathrm{x}$ & $\mathrm{x}$ & $\mathrm{X}$ & \\
\hline Aplicación mortero & & & $\mathrm{x}$ & & & & & & & & & $\mathrm{x}$ & \\
\hline Corte materiales & $\mathrm{X}$ & & $\mathrm{x}$ & $\mathrm{x}$ & $\mathrm{x}$ & & & & & & & $\mathrm{x}$ & \\
\hline Instalación tuberías & & & $\mathrm{x}$ & & & & & & & & & & \\
\hline Piso porcelanato/cerámica & $\mathrm{x}$ & & $\mathrm{x}$ & & & & & & & & & $\mathrm{x}$ & \\
\hline Pintura muros/cielos & & & $\mathrm{x}$ & & & & & & & & & & \\
\hline Carpintería madera/metálica & $\mathrm{X}$ & & $\mathrm{x}$ & & & & & & & & & & \\
\hline Limpieza pisos/muros & & & $\mathrm{X}$ & $\mathrm{x}$ & & & & & & & & & \\
\hline Acopio materiales & & & & & & & & & & $\mathrm{X}$ & & $\mathrm{X}$ & \\
\hline Transportes internos & & $\mathrm{X}$ & & & $\mathrm{X}$ & & & & & & & & $\mathrm{x}$ \\
\hline Transportes externos & & $\mathrm{x}$ & & & & & & & & & & & $\mathrm{X}$ \\
\hline Mantenimiento equipos & & $\mathrm{x}$ & $\mathrm{X}$ & $\mathrm{x}$ & & & & & & & & & \\
\hline Abastecimiento maquinaria & & $\mathrm{x}$ & & & & & & & & & & & $\mathrm{X}$ \\
\hline Pavimento vías & & $\mathrm{x}$ & $\mathrm{X}$ & $\mathrm{X}$ & & & & & & & & $\mathrm{x}$ & $\mathrm{X}$ \\
\hline Arborización & & & & & & $\mathrm{X}$ & $\mathrm{x}$ & $\mathrm{x}$ & $\mathrm{X}$ & & & & \\
\hline Instalación grama & & & & & & $\mathrm{x}$ & & & & & & & \\
\hline Instalación adoquines & $\mathrm{X}$ & $\mathrm{X}$ & $\mathrm{X}$ & $\mathrm{X}$ & & $\mathrm{X}$ & & & & & & $\mathrm{x}$ & \\
\hline
\end{tabular}

Fuente: Elaboración Propia 
SIBRAGEC - ELAGEC 2019 - del 23 al 25 de Octubre - LONDRINA - PR

Continuación Tabla 1

\begin{tabular}{|c|c|c|c|c|c|c|c|c|}
\hline $\begin{array}{l}\text { Impactos } \\
\text { Actividades de } \\
\text { construcción }\end{array}$ & 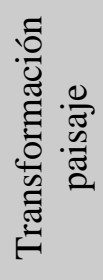 & 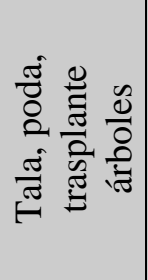 & 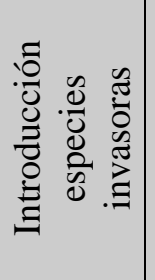 & 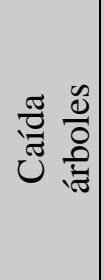 & 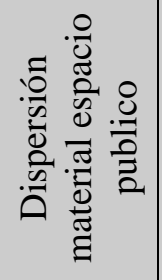 & 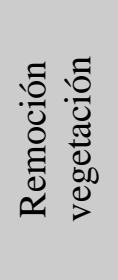 & 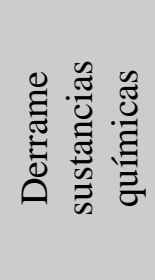 & 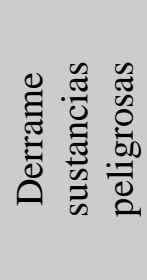 \\
\hline Demolición & & $\mathrm{x}$ & & & & $\mathrm{x}$ & & \\
\hline \multicolumn{9}{|l|}{ Adecuación terreno } \\
\hline Adecuaciones provisionales & & & & & & $\mathrm{x}$ & & \\
\hline Excavación & & $\mathrm{x}$ & & & & & & \\
\hline Descapote vegetación & & & & $\mathrm{x}$ & & & & \\
\hline \multicolumn{9}{|l|}{ Corte/compactación terreno } \\
\hline Uso explosivos & & $\mathrm{x}$ & $\mathrm{x}$ & & & & & \\
\hline Preparación concreto & & $\mathrm{x}$ & $\mathrm{x}$ & & & & & $\mathrm{x}$ \\
\hline \multicolumn{9}{|l|}{ Fundido concreto } \\
\hline \multicolumn{9}{|l|}{ Curado concreto } \\
\hline \multicolumn{9}{|l|}{ Armado encofrado } \\
\hline Impermeabilización & $\mathrm{x}$ & & $\mathrm{x}$ & & & & & $\mathrm{x}$ \\
\hline Preparación mortero & & $\mathrm{x}$ & & & & & & \\
\hline Aplicación mortero & & $\mathrm{x}$ & & & & & & \\
\hline Corte materiales & & $\mathrm{x}$ & & & & & & \\
\hline Instalación tuberías & $\mathrm{x}$ & & $\mathrm{x}$ & & & & & $\mathrm{x}$ \\
\hline Piso porcelanato/cerámica & & $\mathrm{x}$ & & & & & & \\
\hline Pintura muros/cielos & & & $\mathrm{x}$ & & & & & \\
\hline Carpintería madera/metálica & & & & & $\mathrm{x}$ & & & \\
\hline Limpieza pisos/muros & $\mathrm{x}$ & $\mathrm{x}$ & & & & $\mathrm{x}$ & $\mathrm{x}$ & \\
\hline \multicolumn{9}{|l|}{ Acopio materiales } \\
\hline Transportes internos & & & $\mathrm{x}$ & & & & & \\
\hline Transportes externos & & & $\mathrm{x}$ & & & & & \\
\hline Mantenimiento equipos & & & $\mathrm{x}$ & & & $\mathrm{x}$ & & \\
\hline Abastecimiento maquinaria & & & $\mathrm{x}$ & & & & & $\mathrm{x}$ \\
\hline Pavimento vías & $\mathrm{x}$ & & $\mathrm{x}$ & & & & & $\mathrm{x}$ \\
\hline \multicolumn{9}{|l|}{ Arborización } \\
\hline \multicolumn{9}{|l|}{ Instalación grama } \\
\hline Instalación adoquines & & $\mathrm{x}$ & & & & $\mathrm{x}$ & & \\
\hline
\end{tabular}

Fuente: Elaboración Propia

De las actividades más comunes en los procesos constructivos, las 10 que mayores impactos ambientales generan son: preparación de concreto (12), preparación de mortero (9), demolición (8), pavimento de vías (8), instalación de adoquines (8), excavación (7), descapote de vegetación (6), corte/compactación del terreno (6), corte de materiales (6), limpieza de pisos/muros (5). La Gráfica 1, presenta cada actividad y su porcentaje de incidencia sobre los impactos ambientales. A partir de esta grafica es posible establecer acciones que permitan llevar un mejor control de las actividades para finalmente mitigar sus impactos asociados.

Igualmente, pretendiendo identificar aquellos impactos más recurrentes en las actividades de construcción, se elaboró la Gráfica 2, con el propósito de analizar las acciones de mejora e implementar un plan de trabajo para disminuir dichos impactos. 
SIBRAGEC - ELAGEC 2019 - del 23 al 25 de Octubre - LONDRINA - PR

\section{Gráfica 1 - Porcentaje impactos ambientales que genera cada actividad de construcción}

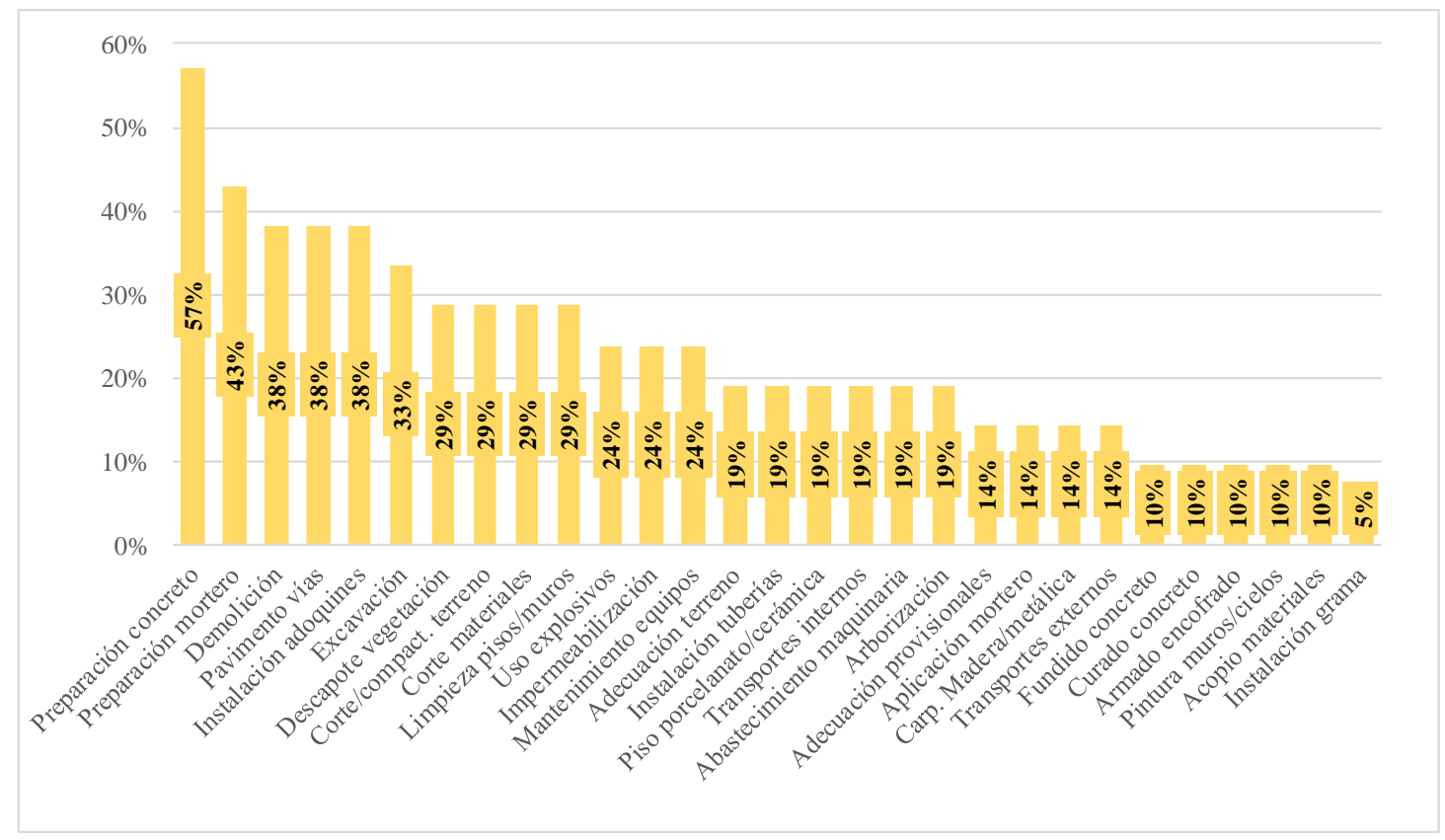

Fuente: Elaboración propia

Gráfica 2 - Porcentaje actividades que compromete cada impacto ambiental

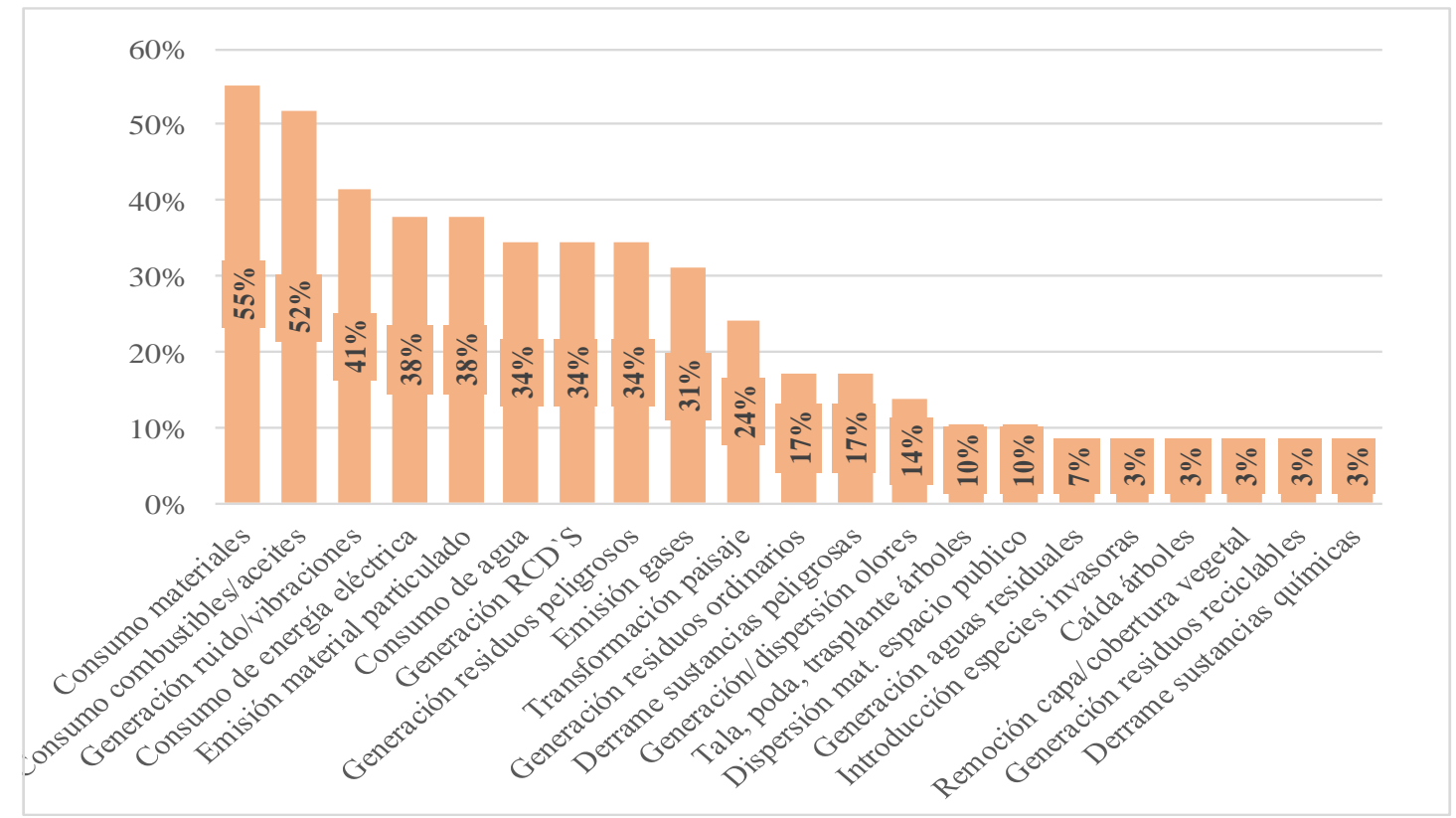

Fuente: Elaboración propia

Los 10 impactos más representativos en las actividades de construcción son: consumo de materiales generado por 16 actividades, consumo de combustibles/aceites (15), generación de ruido/vibraciones (12), consumo de energía eléctrica (11), emisión de material particulado (11), consumo de agua (10), generación de RCDs (10), generación de residuos peligrosos (10), emisión de gases (9) y transformación del paisaje (7) (Gráfica 2). 
SIBRAGEC - ELAGEC 2019 - del 23 al 25 de Octubre - LONDRINA - PR

Tabla 2 - Prácticas Lean que contribuyen a la mitigación o disminución de los impactos ambientales del sector constructor

\begin{tabular}{|c|c|c|c|c|c|c|c|c|c|c|c|c|c|c|c|}
\hline \multirow{2}{*}{\multicolumn{2}{|c|}{$\begin{array}{l}\text { Prácticas } \\
\text { ampactos } \\
\text { Caída árboles }\end{array}$}} & 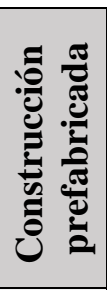 & $\sum$ & 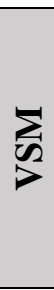 & 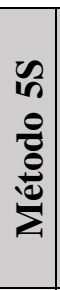 & 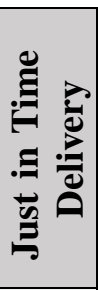 & 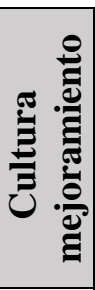 & $\hat{\theta}$ & 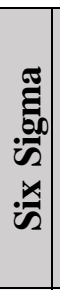 & 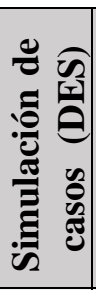 & 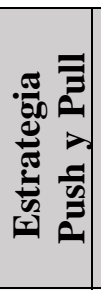 & 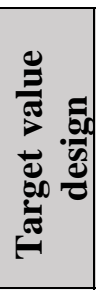 & 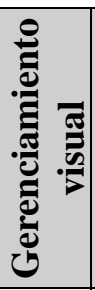 & 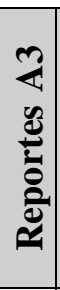 & 㫣 \\
\hline & & & & & & & & & & & & & & & $\mathbf{0}$ \\
\hline \multirow{4}{*}{ 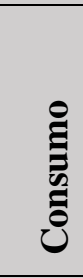 } & Agua & $\mathrm{x}$ & & $\mathrm{x}$ & & & & & & & & & & & 2 \\
\hline & $\begin{array}{l}\text { Combustibles/ } \\
\text { aceites }\end{array}$ & $\mathrm{x}$ & $\mathrm{x}$ & & & & & & & & & & & & 2 \\
\hline & $\begin{array}{l}\text { Energía } \\
\text { eléctrica }\end{array}$ & $\mathrm{x}$ & $\mathrm{X}$ & $\mathrm{x}$ & & $\mathrm{x}$ & & $\mathrm{X}$ & $\mathrm{x}$ & $\mathrm{x}$ & & $\mathrm{x}$ & & & 8 \\
\hline & Materiales & $\mathrm{X}$ & $\mathrm{X}$ & $\mathrm{x}$ & & $\mathrm{x}$ & $\mathrm{x}$ & & $\mathrm{X}$ & & $\mathrm{x}$ & & & & 7 \\
\hline \multirow{2}{*}{ 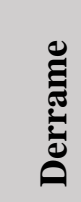 } & $\begin{array}{l}\text { Sustancias } \\
\text { peligrosas }\end{array}$ & & & & & & & & & & & & & & $\mathbf{0}$ \\
\hline & $\begin{array}{l}\text { Sustancias } \\
\text { químicas }\end{array}$ & & & & & & & & & & & & & & $\mathbf{0}$ \\
\hline \multicolumn{2}{|c|}{$\begin{array}{l}\text { Dispersión } \\
\text { materiales espacio } \\
\text { publico }\end{array}$} & & & & & & & & & & & & & & $\mathbf{0}$ \\
\hline \multirow{2}{*}{ 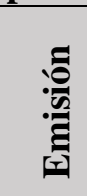 } & $\begin{array}{l}\text { Material } \\
\text { particulado }\end{array}$ & $\mathrm{X}$ & $\mathrm{x}$ & $\mathrm{x}$ & $\mathrm{X}$ & $\mathrm{x}$ & $\mathrm{x}$ & $\mathrm{X}$ & & $\mathrm{x}$ & $\mathrm{x}$ & & & $\mathrm{x}$ & 10 \\
\hline & Gases & $\mathrm{X}$ & $\mathrm{x}$ & $\mathrm{x}$ & $\mathrm{x}$ & $\mathrm{x}$ & $\mathrm{x}$ & $\mathrm{X}$ & & $\mathrm{x}$ & $\mathrm{x}$ & & & $\mathrm{x}$ & 10 \\
\hline \multirow{7}{*}{ 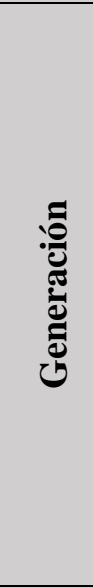 } & $\begin{array}{l}\text { Aguas } \\
\text { residuales }\end{array}$ & $\mathrm{x}$ & & & & & & & & & & & & & 1 \\
\hline & $\begin{array}{l}\text { Ruido/ } \\
\text { vibraciones }\end{array}$ & $\mathrm{X}$ & & & & & & & & & & & & & 1 \\
\hline & $\begin{array}{l}\text { Dispersión } \\
\text { olores }\end{array}$ & & & & & & & & & & & & & & $\mathbf{0}$ \\
\hline & RCD & $\mathrm{x}$ & $\mathrm{x}$ & $\mathrm{x}$ & $\mathrm{x}$ & $\mathrm{x}$ & $\mathrm{x}$ & $\mathrm{X}$ & $\mathrm{x}$ & $\mathrm{x}$ & $\mathrm{x}$ & & $\mathrm{X}$ & $\mathrm{X}$ & 12 \\
\hline & $\begin{array}{l}\text { Residuos } \\
\text { peligrosos }\end{array}$ & $\mathrm{X}$ & $\mathrm{X}$ & $\mathrm{x}$ & $\mathrm{x}$ & $\mathrm{x}$ & $\mathrm{x}$ & $\mathrm{x}$ & $\mathrm{x}$ & $\mathrm{x}$ & $\mathrm{x}$ & & $\mathrm{X}$ & $\mathrm{X}$ & 12 \\
\hline & $\begin{array}{l}\text { Residuos } \\
\text { reciclables }\end{array}$ & $\mathrm{X}$ & $\mathrm{x}$ & $\mathrm{x}$ & $\mathrm{x}$ & $\mathrm{x}$ & $\mathrm{X}$ & $\mathrm{x}$ & $\mathrm{x}$ & $\mathrm{x}$ & $\mathrm{x}$ & & $\mathrm{X}$ & $\mathrm{X}$ & 12 \\
\hline & $\begin{array}{l}\text { Residuos } \\
\text { ordinarios }\end{array}$ & $\mathrm{x}$ & $\mathrm{x}$ & $\mathrm{x}$ & $\mathrm{x}$ & $\mathrm{x}$ & $\mathrm{x}$ & $\mathrm{X}$ & $\mathrm{x}$ & $\mathrm{x}$ & $\mathrm{x}$ & & $\mathrm{x}$ & $\mathrm{X}$ & 12 \\
\hline \multicolumn{2}{|c|}{$\begin{array}{l}\text { Introducción } \\
\text { especies invasoras }\end{array}$} & & & & & & & & & & & & & & $\mathbf{0}$ \\
\hline \multicolumn{2}{|c|}{$\begin{array}{l}\text { Remoción } \\
\text { capa/cobertura } \\
\text { vegetal }\end{array}$} & & & & & & & & & & & & & & 0 \\
\hline \multicolumn{2}{|c|}{$\begin{array}{l}\text { Tala, poda, } \\
\text { trasplante árboles }\end{array}$} & & & & & & & & & & & & & & $\mathbf{0}$ \\
\hline \multicolumn{2}{|c|}{$\begin{array}{l}\text { Transformación } \\
\text { paisaje }\end{array}$} & & & & & & & & & & & & & & 0 \\
\hline & TOTAL & 12 & 9 & 9 & 6 & 8 & 7 & 7 & 6 & 7 & 7 & 1 & 4 & 6 & \\
\hline
\end{tabular}

Fuente: Elaboración Propia con base en el análisis de los estudios que se mencionan en el Anexo. 
SIBRAGEC - ELAGEC 2019 - del 23 al 25 de Octubre - LONDRINA - PR

\subsection{Aporte de las prácticas Lean a la disminución de impactos ambientales en el sector constructor}

LC está centrada en la eliminación de desperdicios para el aumento de la eficiencia, tiempos de entrega y productividad. Estudios han evidenciado que esta filosofía puede aportar a la disminución de los impactos ambientales de la construcción (GHOSH; BHATTACHARJEE; PISHDAD-BOZORGI, 2014; NAHMENS, 2009; SONG; LIANG, 2011), e incluso han relacionado la utilización de prácticas Lean con la mitigación de impactos ambientales durante los procesos de construcción.

En la Tabla 2 se identificaron las prácticas Lean que pueden contribuir a la mitigación de impactos ambientales del sector de la construcción.

De los 21 impactos ambientales identificados en la Tabla 1, 12 son relacionados con prácticas Lean que pueden contribuir a su disminución. Siendo la generación de RCD `S, de residuos ordinarios, reciclables y peligrosos aquellos impactos que mayores oportunidades de mitigación o prevención presentan con la aplicación de prácticas Lean.

Mediante la revisión detallada de la literatura, Tabla 2, se encontraron 13 prácticas Lean que pueden contribuir a la disminución de al menos un impacto ambiental generado por las actividades de construcción consideradas. Según la literatura, la práctica que a más impactos puede aportar es la Construcción prefabricada, contribuyéndole a 12 impactos ambientales. Seguida de BIM y VSM, que pueden contribuir a la disminución de 9 impactos ambientales.

\section{CONCLUSIONES Y CONSIDERACIONES FINALES}

En este estudio se identificaron, cuáles son las actividades de construcción que más impactos generan y cuáles son los impactos más recurrentes en las actividades analizadas. Además, se establecieron 13 prácticas de LC que pueden contribuir a la disminución o prevención de algunos de los impactos ambientales encontrados.

Hay algunos impactos que no se encuentran relacionados con prácticas Lean, esto no precisamente representa que no haya herramientas que puedan aportar a la disminución de estos, sino que no han sido lo suficientemente estudiados. Asimismo, existen prácticas Lean que mediante este estudio no se encontraron como contribuyentes a la disminución o mitigación de impactos ambientales. Lo anterior deja abierto el panorama para futuros estudios.

\section{REFERENCIAS}

$360^{\circ}$ EN CONCRETO. Caracterización de impactos ambientales en la industria de la construcciónBlog $360^{\circ}$ en concreto. [s.l: s.n.]. Disponível em:

$<$ http://blog.360gradosenconcreto.com/caracterizacion-de-impactos-ambientales-en-laindustria-de-la-construccion/>.

AHUJA, R. Lean and Green Construction. International Journal of Scientific \& Engineering Research, v. 3, n. 7, p. 1-4, 2012.

AHUJA, R. Sustainable Construction: Is Lean Green? ICSDEC 2012, p. 903-911, 2013.

AHUJA, R.; SAWHNEY, A.; ARIF, M. Driving lean and green project outcomes using BIM : A qualitative comparative analysis. International Journal of Sustainable Built Environment, v. 6, n. 1, p. 69-80, 2017. 
AJAYI, S. O. et al. Critical management practices influencing on-site waste minimization in construction projects. Waste Management, v. 59, p. 330-339, 2017.

ALIA, A. et al. The path towards greening the Malaysian construction industry. v. 52, p. 17421748, 2015.

ALWAN, Z.; JONES, P.; HOLGATE, P. Strategic sustainable development in the UK construction industry, through the framework for strategic sustainable development, using Building Information Modelling. Journal of Cleaner Production, v. 140, p. 349-358, 2017.

AMBIENTE, S. D. DE. Guía de Manejo Ambiental para la Construcción, Segunda edición. [s.l: s.n.]. Disponível em:

<http://ambientebogota.gov.co/documents/664482/0/GUIA_MANEJO_AMBIENTAL_FINAL. pdf $>$.

BAE, J.-W.; KIM, Y.-W. Sustainable value on construction project and application of lean construction methods. Lean Construction: A New Paradigm for Managing Capital Projects - 15th IGLC Conference, n. July, p. 312-321, 2007.

BANAWI, A.; BILEC, M. M. A framework to improve construction processes: Integrating lean, green and six sigma. International Journal of Construction Management, v. 14, n. 1, p. 45$55,2014$.

BRE ENVIRONMENTAL CONSULTANCY. Sustainable construction Simple ways to make it happen. [s.l: s.n.].

CALDERÓN, J. J. T. Analisis Constructivo del proceso de evaluación de impacto ambiental en Colombia, propuestas de mejora. [s.l: s.n.].

$\mathrm{CAO}, \mathrm{X}$. et al. A comparative study of environmental performance between prefabricated and traditional residential buildings in China. Journal of Cleaner Production, v. 109, p. 131-143, 2015.

CARNEIRO, S. B. M. et al. LEAN and green: A relationship matrix. 20th Conference of the International Group for Lean Construction, IGLC 2012, July 17, 2012 - July 22, 2012, 2012.

CHEN, Y.; OKUDAN, G. E.; RILEY, D. R. Automation in Construction Sustainable performance criteria for construction method selection in concrete buildings. Automation in Construction, v. 19, n. 2, p. 235-244, 2010.

CHENG, S. et al. Durability and environment evaluation of an eco-friendly cement-based material incorporating recycled chromium containing slag. Journal of Cleaner Production, v. 185, p. 23-31, 2018.

CHONG, H.; LEE, C.; WANG, X. A mixed review of the adoption of Building Information Modelling ( BIM ) for sustainability. Journal of Cleaner Production, v. 142, p. 4114-4126, 2017.

CHU, J. Solutions to sustainability in construction : some examples. Procedia Engineering, v. 145, p. 1127-1134, 2016.

DE CARVALHO, A. C. V.; GRANJA, A. D.; DA SILVA, V. G. A systematic literature review on integrative lean and sustainability synergies over a building's lifecycle. Sustainability (Switzerland), v. 9, n. 7, 2017.

DHINGRA, R.; KRESS, R.; UPRETI, G. Does lean mean green ? Journal of Cleaner Production, v. 85, p. 1-7, 2014.

ENSHASSI, A.; KOCHENDOERFER, B.; RIZQ, E. An evaluation of environmental impacts of construction projects. Revista Ingenieria de Construccion, v. 29, n. 3, p. 234-254, 2014. 
EPA. Pursuing Perfection: Case Studies Examining Lean Manufacturing Strategies, Pollution Prevention, and Environmental Regulatory Management Implications. Michigan health \& hospitals, v. 38, n. 4, p. 22-23, 2000.

FIRMAWAN, F.; OTHMAN, F.; YAHYA, K. Improving project performance and waste reduction in construction projects: A case study of a Goverment institutional building project. International Journal of Technology, p. 182-192, 2012.

FUENZALIDA, C.; ARROYO, P.; SALVATIERRA, J. L. Evaluating Environmental Impacts of Construction Operation Before and After the Implementation of. International Group for Lean Construction, n. January, p. 3-12, 2016.

GANIYU, B. O.; FAPOHUNDA, J. A.; HALDENWANG, R. Construction Approaches to Enhance Sustainability in Affordable Housing in Developing Countries. World Congress on Sustainable Technologies. Anais...2015

GHOSH, S.; BHATTACHARJEE, S.; PISHDAD-BOZORGI, P. A Case Study To Examine Environmental Benefits of Lean. Proceedings IGLC-22,. Anais...2014

GOLZARPOOR, H. et al. An input-output simulation model for assessing production and environmental waste in construction. Journal of Cleaner Production, v. 143, p. 1094-1104, 2017.

GOLZARPOOR, H.; GONZÁLEZ, V. A green-Lean simulation model for assessing environmental and production waste in construction. Iglc-21, p. 885-894, 2013.

GONZÁLEZ, J. A. A. Manual de evaluación de impacto ambiental de proyectos, obras o actividades. [s.l: s.n.].

JAILLON, L.; POON, C. S.; CHIANG, Y. H. Quantifying the waste reduction potential of using prefabrication in building construction in Hong Kong. Waste Management, v. 29, n. 1, p. 309-320, 2009.

JOHNSEN, C. A.; DREVLAND, F. Lean and Sustainability: Three Pillar Thinking in the Production Process. International Group for Lean Construction, n. 23, p. 23-32, 2016.

KAMALI, M.; HEWAGE, K. Life cycle performance of modular buildings : A critical review. Renewable and Sustainable Energy Reviews, v. 62, p. 1171-1183, 2016.

KAMALI, M.; HEWAGE, K. Development of performance criteria for sustainability evaluation of modular versus conventional construction methods. Journal of Cleaner Production, v. 142, p. 3592-3606, 2017.

KHODEIR, L. M.; OTHMAN, R. Examining the interaction between lean and sustainability principles in the management process of AEC industry. Ain Shams Engineering Journal, 2016.

LI, S. et al. A study on the evaluation of implementation level of lean construction in two Chinese fi rms. Renewable and Sustainable Energy Reviews, v. 71, n. January 2016, p. 846851, 2017.

M. S. BAJJOU, A. CHAFI, A. E. AND M. E. H. The Practical Relationships between Lean Construction Tools and Sustainable Development: A literature review. Journal of Engineering Science and Technology Review, v. 10, n. 4, p. 170-177, 2017.

MARIS, K.; PARRISH, K. The Confluence of Lean and Green Construction Practices in the comercial buildings market. Proc. 24th Ann. Conf. of the Int'1. Group for Lean Construction. Anais...2016

MARJABA, G. E.; CHIDIAC, S. E. Sustainability and resiliency metrics for buildings e Critical review. Building and Environment, v. 101, p. 116-125, 2016.

NAHMENS, I. From Lean to Green Construction: A natural extension. Construction Research Congress 2009. Anais...2009 
NASCIMENTO, D. et al. Digital Obeya Room : exploring the synergies between BIM and lean for visual construction management. Innovative Infrastructure Solutions, p. 1-10, 2018.

OGUNBIYI, O.; OLADAPO, A.; GOULDING, J. An empirical study of the impact of lean construction techniques on sustainable construction in the UK. Construction Innovation, v. 14, n. 1, p. 88-107, 2014.

PARRISH, K. Lean and Green Construction: Lessons learned from design and construction of a modular LEED Gold Building. Proceedings for the 20th Annual Conference of the International Group for Lean Construction. Anais...2012

PULASKI, M. et al. Synergies between Sustainable Design and Constructability at the Pentagon. p. 1-8, 2004.

RAY, H.; EL, A.; GILBERT, B. P. A comparative life cycle study of alternative materials for Australian multi-storey apartment building frame constructions : Environmental and economic perspective. Journal of Cleaner Production, v. 166, p. 458-473, 2017.

ROSENBAUM, S.; TOLEDO, M.; GONZALEZ, V. Green-Lean Approach for assessing Environmental and Production Waste in Construction. Proceedings for the 20th Annual Conference of the International Group for Lean Construction. Anais...2008

ROSENBAUM, S.; TOLEDO, M.; GONZÁLEZ, V. Improving Environmental and Production Performance in Construction Projects Using Value-Stream Mapping: Case St. Journal of construction engineering and management, v. 140, 2014.

SAGGIN, A. B. et al. Comparing Investments in Sustainability With Cost Reduction From Waste Due to Lean Construction. 23rd Annual Conference of the International Group for Lean Construction, p. 223-230, 2015.

SAIEG, P. et al. Interactions of Building Information Modeling, Lean and Sustainability on the Architectural, Engineering and Construction industry: A systematic review. Journal of Cleaner Production, v. 174, p. 788-806, 2018.

SALEM, O. et al. Reducing environmental, economic, and social impacts of work-zones by implementing lean construction techniques. 22nd Annual Conference of the International Group for Lean Construction: Understanding and Improving Project Based Production, IGLC 2014, v. 1, n. 315, p. 145-155, 2014.

SALGIN, B.; ARROYO, P.; BALLARD, G. Exploring the relationship between lean design methods and C \& D waste reduction : three case studies of hospital projects in California Explorando la relación entre los métodos de diseño lean y la reducción de residuos de construcción y demolición : tres. v. 31, p. 191-200, 2016.

SAPUAY, S. E. Construction Waste - Potentials and Constraints. Procedia Environmental Sciences, v. 35, p. 714-722, 2016.

SONG, L.; LIANG, D. Lean construction implementation and its implication on sustainability: a contractor's case study. Canadian Journal of Civil Engineering, v. 38, n. 3, p. 350-359, 2011.

TAM, V. W. Y. et al. Best practice of prefabrication implementation in the Hong Kong public and private sectors. Journal of Cleaner Production, v. 109, p. 216-231, 2015.

VALENTE, C. P.; MOURÃO, C. A. M. DO A.; BARROS NETO, J. D. P. Lean and Green : How Both Philosophies Can Interact on Strategic , Tactical and Operational Levels of a Company. Proceeding of 201h Annual Conference of IGLC, n. July, p. 925-934, 2013.

VASCONCELOS, I. A. DE et al. Guidelines for Practice and Evaluation of Sustainable Construction Sites : a Lean, Green and Wellbeing Integrated Approach. Annual Conference of the IGLC, n. November, p. 773- 782., 2015. 


\section{SIBRAGEC - ELAGEC 2019 - del 23 al 25 de Octubre - LONDRINA - PR}

VAZQUEZ, E. et al. Sustainability in civil construction applied in the construction site phase. v. 144, p. 265-276, 2011.

VERRIER, B.; ROSE, B.; CAILLAUD, E. Lean and Green strategy : the Lean and Green House and maturity deployment model. Journal of Cleaner Production, v. 116, p. 150-156, 2016.

VIEIRA, A. R.; CACHADINHA, N. Lean construction and sustainability-complementary paradigms-a case study. IGLC-19. Anais...2011

VILLORIA, P. et al. Best practice measures assessment for construction and demolition waste management in building constructions. "Resources, Conservation \& Recycling", v. 75, p. 5262, 2013.

WANG, J.; LI, Z.; TAM, V. W. Y. Identifying best design strategies for construction waste minimization. Journal of Cleaner Production, v. 92, p. 237-247, 2015.

WEINHEIMER, N. The Process of Green Building Certification: An Examination regarding Lean Principles. Proc. 24th Ann. Conf. of the Int'1. Group for Lean Construction. Anais...2016

WEINHEIMER, N.; SCHMALZ, S.; MÜLLER, D. Green Building and Lean Management : Synergies and Conflicts. v. II, n. July, p. 911-918, 2017.

WING, T. Lean-based clean earthworks operation. v. 142, 2017.

$\mathrm{ZHU}, \mathrm{H}$. et al. The exploration of the life-cycle energy saving potential for using prefabrication in residential buildings in China. Energy \& Buildings, v. 0, p. 1-10, 2018.

\section{ANEXO}

Fuentes de la Tabla 2: AHUJA, 2012, 2013; AHUJA; SAWHNEY; ARIF, 2017; AJAYI et al., 2017; ALIA et al., 2015; ALWAN; JONES; HOLGATE, 2017; BAE; KIM, 2007; BANAWI; BILEC, 2014; BRE ENVIRONMENTAL CONSULTANCY, 2008; CAO et al., 2015; CHEN; OKUDAN; RILEY, 2010; CHENG et al., 2018; CHONG; LEE; WANG, 2017; CHU, 2016; DE CARVALHO; GRANJA; DA SILVA, 2017; DHINGRA; KRESS; UPRETI, 2014; FIRMAWAN; OTHMAN; YAHYA, 2012; FUENZALIDA; ARROYO; SALVATIERRA, 2016; GANIYU; FAPOHUNDA; HALDENWANG, 2015; GHOSH; BHATTACHARJEE; PISHDAD-BOZORGI, 2014; GOLZARPOOR et al., 2017; GOLZARPOOR; GONZÁLEZ, 2013; JAILLON; POON; CHIANG, 2009; JOHNSEN; DREVLAND, 2016; KAMALI; HEWAGE, 2016, 2017; KHODEIR; OTHMAN, 2016; LI et al., 2017; M. S. BAJJOU, A. CHAFI, 2017; MARIS; PARRISH, 2016; MARJABA; CHIDIAC, 2016; NAHMENS, 2009; NASCIMENTO et al., 2018; OGUNBIYI; OLADAPO; GOULDING, 2014; PARRISH, 2012; RAY; EL; GILBERT, 2017; ROSENBAUM; TOLEDO; GONZALEZ, 2008; ROSENBAUM; TOLEDO; GONZÁLEZ, 2014; SAIEG et al., 2018; SALEM et al., 2014; SALGIN; ARROYO; BALLARD, 2016; SAPUAY, 2016; SONG; LIANG, 2011; TAM et al., 2015; VALENTE; MOURÃO; BARROS NETO, 2013; VAZQUEZ et al., 2011; VERRIER; ROSE; CAILLAUD, 2016; VIEIRA; CACHADINHA, 2011; VILLORIA et al., 2013; WANG; LI; TAM, 2015; WEINHEIMER, 2016; WEINHEIMER; SCHMALZ; MÜLLER, 2017; WING, 2017; ZHU et al., 2018. 